http://jmscr.igmpublication.org/home/ ISSN (e)-2347-176x ISSN (p) 2455-0450

crossref DOI: https://dx.doi.org/10.18535/jmscr/v8i2.19

\title{
Combination of Modified Alvarado Score and Ultrasonography to Improve the Diagnostic Accuracy in Acute Appendicitis
}

\author{
Authors \\ Dr Talagadadeevi Dheeraj ${ }^{1}$, Dr G Sumanth², Dr V Devi Harsha ${ }^{3}$, \\ Dr M Sai Harish ${ }^{4}$, Dr V Tatha Rao ${ }^{5}$ \\ ${ }^{1,2,3,4}$ Post Graduate, Department of General Surgery \\ ${ }^{5}$ Professor of General Surgery
}

\begin{abstract}
Background: Acute appendicitis, the most common cause of pain abdomen in emergency settings. The diagnosis of acute appendicitis can most often pose a difficulty.

Methods: Hundred consecutive patients who presented with symptoms consistent with the diagnosis of acute appendicitis and were admitted in the department of surgery, NRI Academy of Sciences, Guntur, Andhra Pradesh, have been taken into this study. The patients were evaluated prospectively with the modified Alvarado scoring (MAS), and the possibility of acute appendicitis is determined. The Modified Alvarado scores were correlated with Ultrasound and histopathological findings after surgery.

Results: There were 75 true positives and 16 false-negative cases with modified Alvarado scoring. There were 84 true positives and 7 false-negative cases with ultrasound abdomen.

Conclusions: The Modified Alvarado score should be combined with Ultrasound abdomen for a better diagnosis of acute appendicitis. However, these only act as an adjunct to clinical evaluation and the surgeon's experience.
\end{abstract}

Keywords: Modified Alvarado Score (MAS), Appendicitis, Ultrasound abdomen, true positive, false negative.

\section{Introduction}

Acute appendicitis remains the most common abdominal emergencies, demanding surgical intervention. The mortality rate has decreased since the advent of antibiotics. Usually, the picture of appendicitis is often not classical, so the diagnostic process takes longer, thus delaying surgery, increasing the possibility of complications. On the other hand, operation without an accurate diagnosis will lead to negative appendectomy, increasing the morbidity ${ }^{1,2}$.

Even with the modern diagnostic tools, misdiagnosis of appendicitis has remained constant. Diagnostic approaches include symptoms, physical examinations, laboratory findings, and imaging modalities like ultrasonography and computerized tomography (CT) of the abdomen. Although the ultrasound has improved the diagnosis of appendicitis, it is highly operator dependent. The abdominal CT carries the risk of radiation exposure and also increases the cost of treatment ${ }^{3}$. Most surgeons tend to rely on an abdominal ultrasound followed by $\mathrm{CT}$ for objective diagnosis.

As quoted by Bailey \& Love, "Notwithstanding advances in modern radiographic imaging and 
diagnostic laboratory investigations, the diagnosis of appendicitis remains essentially clinical, requiring a mixture of observation, clinical acumen, and surgical science" ${ }^{4}$.

Many scoring systems have been designed for the diagnosis of acute appendicitis. Among those systems, the Alvarado system is simple to apply and efficient ${ }^{5,6}$. The Alvarado score system has eight variables, with a total score of ten points.

\section{Aims and Objectives}

$>$ To evaluate the role of Ultrasound in the diagnosis of acute appendicitis in clinically equivocal cases

To evaluate the role of modified Alvarado score in the diagnosis of acute appendicitis

To determine the diagnostic accuracy of combined use of ultrasound and modified Alvarado scoring system

\section{Material and Methods \\ Study Design}

Non-randomized prospective study design.

Study Duration

Two years (August 2017 to July 2019).

\section{Study Place}

NRI Academy of Sciences, Guntur, Andhra Pradesh.

\section{Study Population}

Patients presenting with pain right lower quadrant of the Abdomen, lasting fewer than seven days after clinical examination, were provisionally diagnosed to have acute appendicitis.

\section{Sample Size}

One hundred patients reporting to the Surgery department within study duration and eligible as per inclusion criteria will be included in the study.

\section{Inclusion Criteria}

Patients with the provisional clinical diagnosis of acute appendicitis

\section{Exclusion Criteria}

1) Patients of age $<12$ years

2) Patients with generalized peritonitis due to appendicular perforation

3) Patients with appendicular mass or abscess

\section{Data Collection}

First, 100 patients who presented with pain in the right lower quadrant of abdomen who after examination were provisionally diagnosed to have appendicitis were evaluated in this study. After detailed examination and investigations, a modified Alvarado score was applied to cases in the study. The ultrasound findings and the modified Alvarado score findings were compared to the histopathological findings after surgery. The statistical significance of both modalities was obtained and compared.

\section{Results}

Sex Distribution: There were 62 male patients and 38 female patients among the 100 patients studied

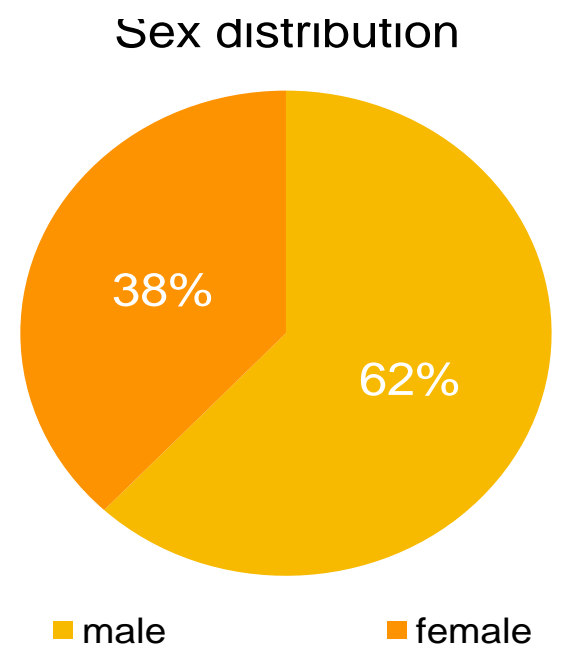




\section{JMSCR Vol||08||Issue ||02||Page 90-96||February}

Age and Sex Distribution: There were 23 male patients and 18 female patients under 18 years of age, and there were 39 male patients and 20 female patients above 18 years of age.

\section{Age and sex distribution}

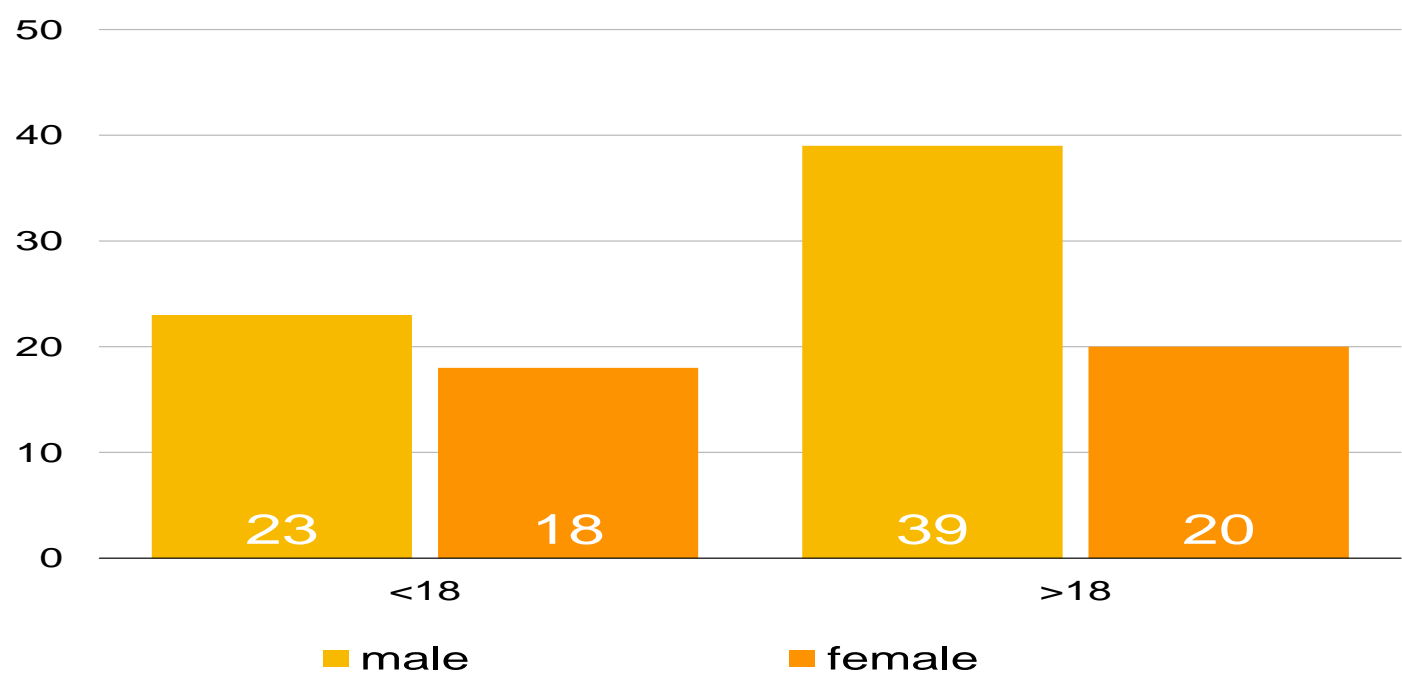

\section{Modified Alvarado Score and Histopathological Correlation}

75 out of 100 patients had Alvarado score more than 7 and 25 patients had a score less than 7

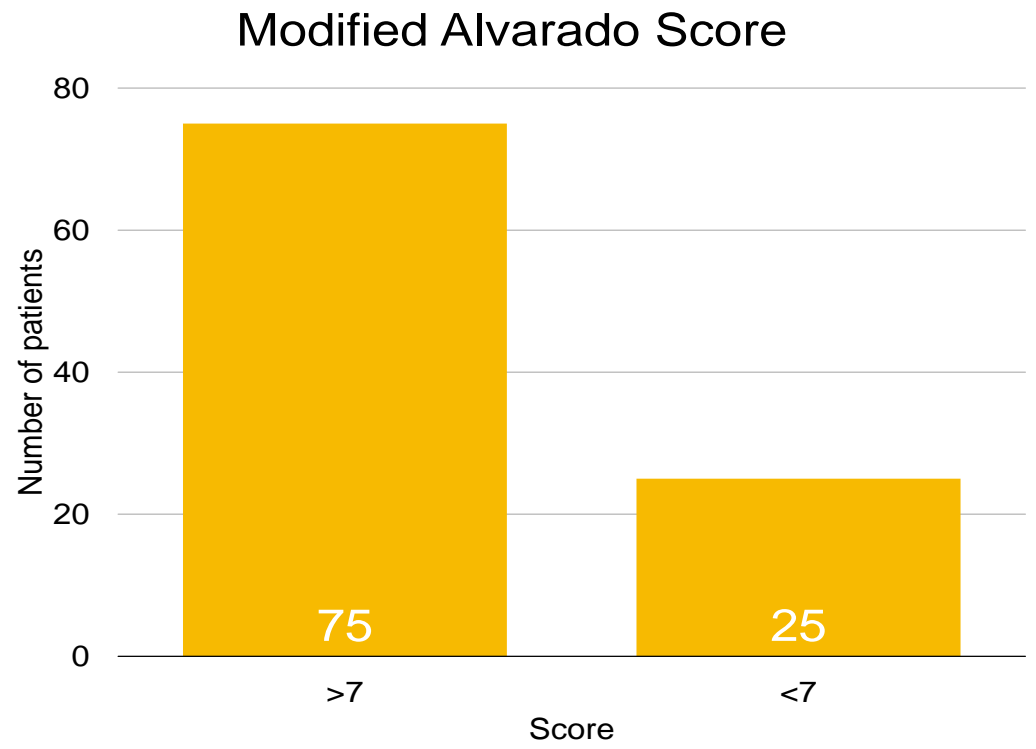

There were 91 cases with positive histopathological features of appendicitis and 9 negative appendicectomies. 


\section{HISTOPATHOLOGY}

100
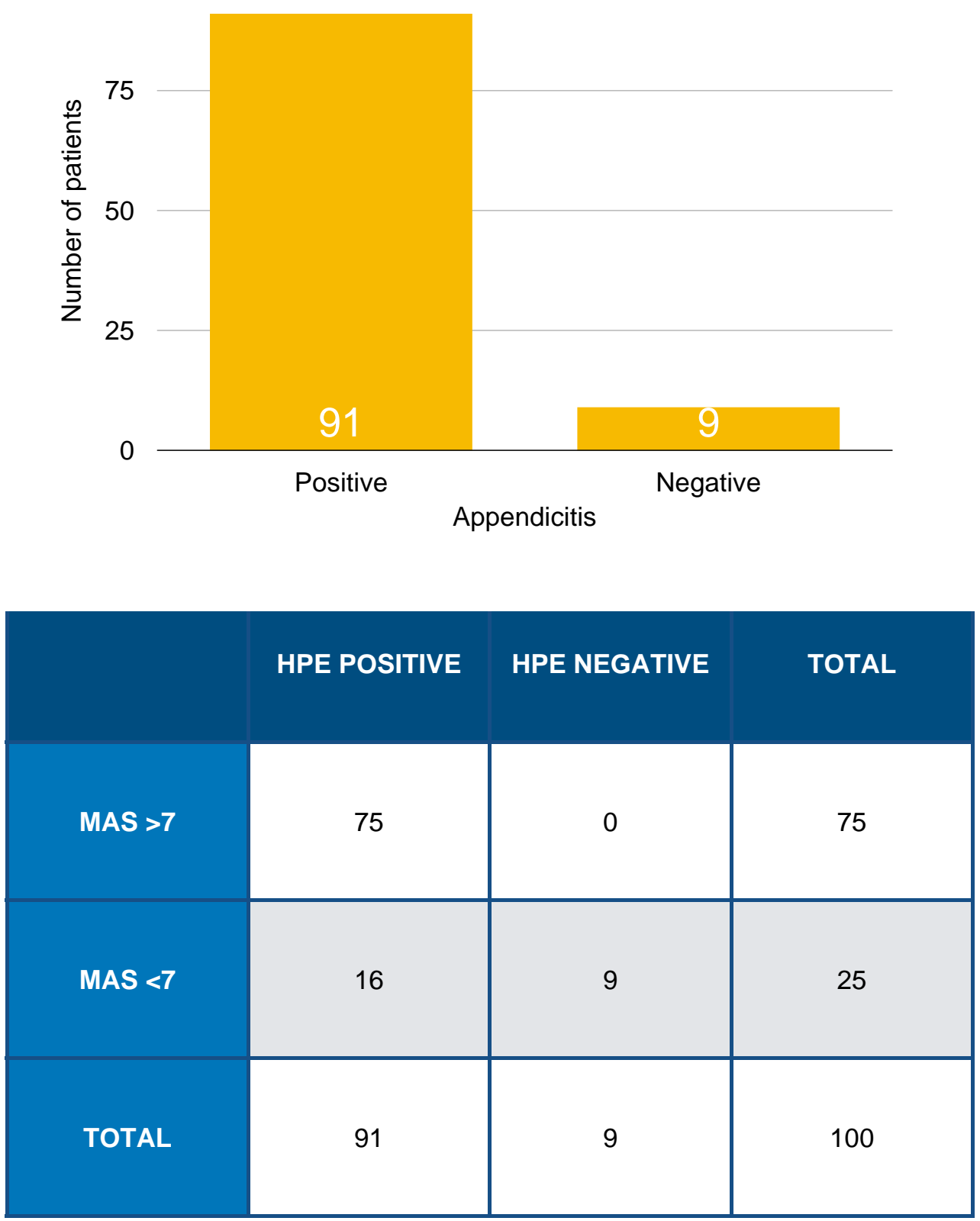

75 cases who had a MAS score of $>7$ were found to have histopathological features positive for appendicitis: True positive

None of the cases with MAS > 7 were negative for histopathological findings of appendicitis: False positive

16 cases with MAS $<7$ were found to have histopathological features positive for appendicitis: False negative

9 cases with MAS $<7$ were negative for histopathological findings of appendicitis: True negative
The sensitivity of MAS in diagnosing acute appendicitis: $82.42 \%$

The specificity of MAS: $100 \%$

Positive predictive value: $100 \%$

Negative predictive value: $36 \%$

Ultrasound and Histopathological Correlation

Of the 100 cases, 84 cases had Ultrasound features of acute appendicitis. 

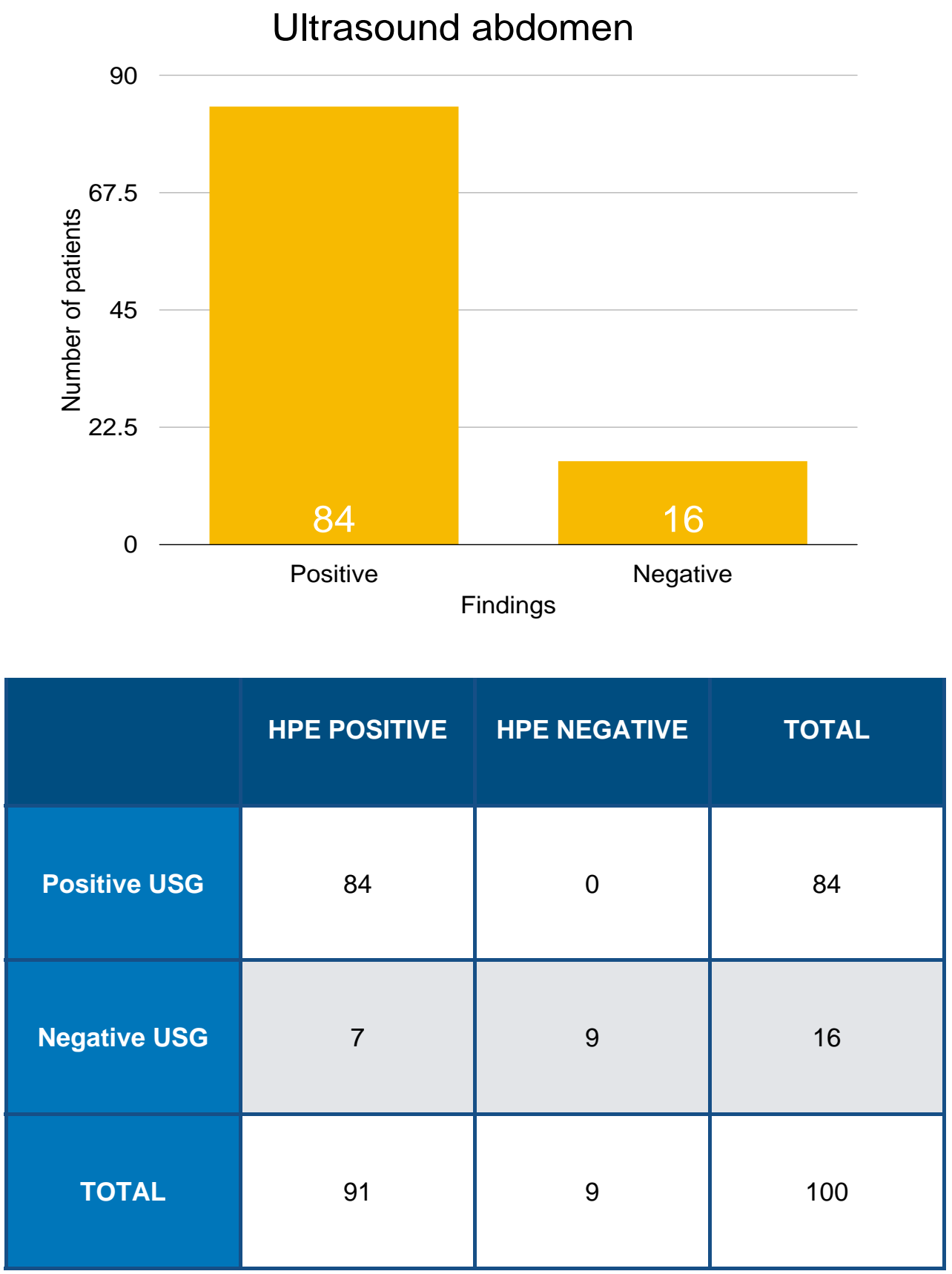

84 cases who had Ultrasound features of appendicitis were found to have histopathological features positive for appendicitis: True positive None of the cases with positive Ultrasound features were negative for histopathological findings of appendicitis: False positive

7 cases with negative/no Ultrasound features of appendicitis were found to have histopathological features positive for appendicitis: False negative
9 cases with negative/no Ultrasound features of appendicitis were negative for histopathological findings of appendicitis: True negative

The sensitivity of USG in diagnosing acute appendicitis: $92.31 \%$

The specificity of USG: $100 \%$

Positive predictive value: $100 \%$

Negative predictive value: $56.25 \%$ 
Comparison between Modified Alvarado Scoring and Ultrasound Abdomen in Diagnosing Acute Appendicitis

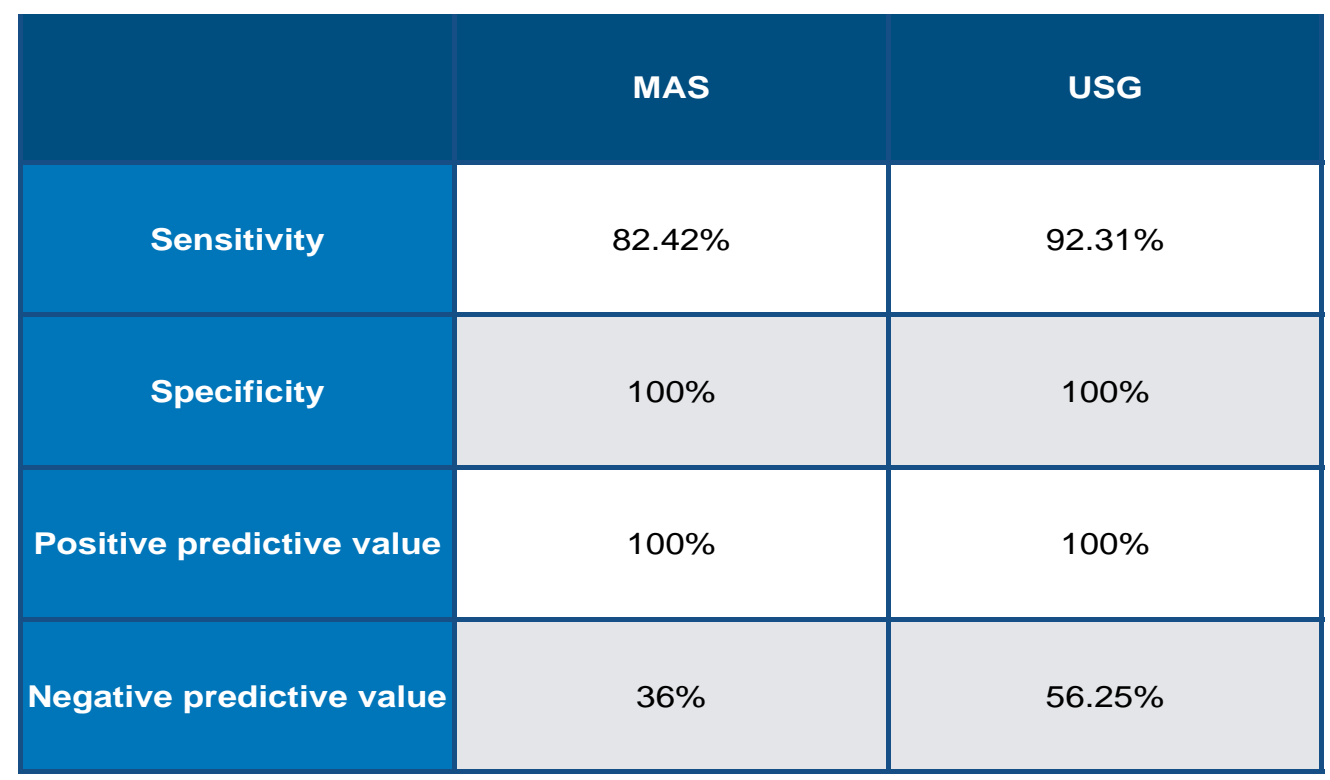

The sensitivity of ultrasound $(92.31 \%)$ is more than the modified Alvarado score $(82.42 \%)$ in diagnosing acute appendicitis.

Both ultrasound and modified Alvarado score are $100 \%$ specific for acute appendicitis.

Both have a positive predictive value of $100 \%$

The ultrasound had negative predictive value of $56.25 \%$, which is more than that of the modified Alvarado score, $36 \%$.

\section{Discussion}

Alvarado is the most commonly known scoring system. Misdiagnosis or delayed diagnosis are the common pitfalls of the Alvarado scoring system. In1986, Alvarado score was first reported based on the review of patients who had undergone appendectomy with suspicion of acute appendicitis. Other than the Alvarado score, many scoring systems had been developed, but the outcome did not differ much, these scoring systems never enjoyed wide application because of their suboptimal discriminatory property.

Management strategy in suspected patients of appendicitis remains a challenge even after the introduction of USG, CT. Usually, USG and CT are used in suspected patients of acute appendicitis. CT is being used optimally to minimize exposure to ionizing radiation. False- negative results may delay surgery and increase morbidity. Complicated appendicitis was more common in males than females, mainly due to the high pain threshold leading to late presentation. Smoking might be a cause due to vascular disease associated with smoking, "something that needs further study.

Normal appendix, according to histopathology after an appendectomy, was common in females than males most probably due to low pain threshold in females and due to the presence of other gynecological causes of lower abdominal pain like pelvic inflammatory disease or tuboovarian causes.

Decisions to operate based solely on clinical examination, result in a higher rate of negative appendectomies. Negative appendectomy leads to severe morbidity and even mortality.

This study is a trial to improve the diagnostic accuracy by using a combination of modified Alvarado score and ultrasonography in acute appendicitis at NRI Academy of Sciences, Guntur, Andhra Pradesh.

In this study, the Modified Alvarado score (MAS) of 7 and above had a positive predictive value of $100 \% .75 \%$ of the cases are true positive with MAS 7 or above and positive histopathology features of acute appendicitis. This gave a crude 
negative appendicectomy rate of $12 \%$ that is in keeping with what Ongaro ${ }^{7}$ found in his study in the 2007 Year. A systematic review by Ohle et $\mathrm{al}^{8}$. found out that a high Alvarado score was less sensitive as a 'rule in' score than as a 'rule out' for those below score 6 . Our study suggests that a high Alvarado score is a useful tool to set aside patients for immediate appendicectomy without further diagnostics. This is in contrast with studies by Saidi and Chavda ${ }^{9}$, that suggested that the scoring system has no value over clinical acumen. In this study, 84 cases(true positive) who had Ultrasound features of appendicitis were found to have histopathological features positive for appendicitis, 7 cases with negative/no Ultrasound features of appendicitis were found to have histopathological features positive for appendicitis (false negative). The sensitivity of USG in diagnosing acute appendicitis: $92.31 \%$. The additional information by ultrasonography may reduce the diagnostic dilemmas in acute appendicitis. The utility of ultrasound has been supported in numerous studies both as an adjunct to improve diagnosis in the equivocal cases and to determine who needs further imaging with a superior modality. In a study by Rasoul, et al. in Iran, ultrasonography had a PPV of $90.4 \%$ and a sensitivity of $55.4 \%$. This is very less and suboptimal when compared to the present study. Kimaro $^{10}$, radiology resident in 2011 did a study on the correlation of ultrasonography as compared to clinical and surgical findings in pain abdomen. His study revealed sensitivity, specificity, PPV and NPV values of $92 \%, 58.3 \%, 95 \%$ and $47 \%$ respectively. The findings of this study were similar to our study. However, ultrasound is operator dependent, and its significance varies among the studies.

\section{Conclusion}

The combination of modified Alvarado score to the ultrasound improves the diagnostic accuracy in appendicitis. However, clinical examination and experience of the clinician are more important than the scoring systems and radiological investigations.

\section{References}

1. Flum DR, Koepsell T. The clinical and economic correlates of misdiagnosed appendicitis: nationwide analysis. Arch Surg 2002, 137(7):799-804.

2. Blomqvist PG, Andersson RE, Granath F, Lambe MP, Ekbom AR. Mortality after appendectomy in Sweden, 1987-1996. Ann Surg 2001, 233(4):455-60.

3. Hall EJ. Cancer risks from diagnostic radiology. Br J Radiol 2008, 81(965):362-78.

4. Hamilton Bailey's "Emergency Surgeries," $12^{\text {th }} \mathrm{Ed}, 1995 ; 438-451$

5. Alvarado A. A practical score for the early diagnosis of acute appendicitis.Ann Emerg Med. 1986; 15:557-64.

6. Douglas CD, Macpherson NE, Davidson PM. Randomised controlled trial of ultrasonography in the diagnosis of acute appendicitis, incorporating the Alvarado score. BMJ. 2000; 321(7266):919-22.

7. Ongaro, Neford. Evaluation of the usefulness of modified Alvarado scoring system regarding early diagnosis of acute appendicitis and in reduction of negative appendicectomy at Kenyatta National Hospital. A prospective study. M med dissertation, Dept of Surgery, University of Nairobi 2005.

8. Ohle, Robert., O'Reilly, F., O'Brien, K., K., et al. The Alvarado score for predicting acute appendicitis: a systematic review. BMC Medicine, 2011, 9:139.

9. Saidi, H.5., Chavda, S.K., Use of a modified Alvarado score in the diagnosis of acute appendicitis. East Afr Med J. 2003 Aug; 80(8):411-414.

10. Kimaro, S. Correlation of ultrasound, clinical, and surgical findings of suspected acute appendicitis in KNH. MMed dissertation. University' of Nairobi 2011. 